\title{
Disappearance of Temporal Collinearity in Vertebrates and its eventual reappearance
}

\section{Spyros Papageorgiou}

NCSR 'Demokritos', Athens, Greece

\begin{abstract}
1. Introduction

Hox Gene Collinearity (HGC) is a fundamental property controlling development particularly in the early embryonic stages of vertebrates and many other animal phyla. E.B. Lewis was the first who observed this phenomenon in the Drosophila $B X-C$ gene complex [1]. Lewis noticed that a class of genes (later denoted as Hox genes) are located in an ordered sequence (Hox1, Hox2, Hox3 ...) along the direction 3' to 5' on the genome DNA. These genes are expressed in the same order in the embryo along the Anterior/Posterior axis. This common order in the chromosome and the embryo is denoted spatial collinearity (SC) [1, 2 ]. SC is a strange property correlating entities at the macroscopic scale of the embryo (of the order of $1 \mathrm{~mm}$ ) and the microscopic scale of the chromosome (of the order of $500 \mathrm{~nm}$ ). This multiscale correlation (extending to about 4 orders of magnitude) is characteristic of Systems Biology [3]. Besides SC it was later observed, particularly in vertebrates, a temporal collinearity (TC). According to TC, the sequence (Hox1, Hox $2, \operatorname{Hox} 3, \ldots)$ are activated in a time order: first Hox1 is activated in ontogenetic unit 1, followed later by Hox 2 in unit 2, and even later by Hox3,... [4].

In 1999 T. Kondo and D. Duboule (K-D) published a remarkable article describing several experiments performed in Duboule's Laboratory [5]. K-D examined the regulatory region of vertebrate Hox gene clusters and in particular they investigated the posterior upstream DNA domain of the mouse HoxD cluster. They excised upstream domains of DNA of variable length up to an extremely large upstream domain cut-off (including Evx gene), and they tested the time of expression of Hoxd4 and Hod10 which lie anteriorily of the extended deleted region. K-D noticed that Hoxd4 and Hoxd10 are very prematurely expressed 'at a time corresponding to that of Hoxd1 'as if' temporal collinearity had disappeared' [5] (I).
\end{abstract}

Here is followed the 'prediction in retrospect' of a Biophysical Model (BM) and it is assumed that TC disappeared indeed.

\section{BM formulation and its elastic spring approximation}

The biophysical model (BM) was formulated after the K-D experiments. According to BM, physical forces are created at the telomeric end of the Hox cluster pulling sequentially the Hox genes towards a transcription factory domain where genes are transcribed [6,7] (Fig.1). At the 


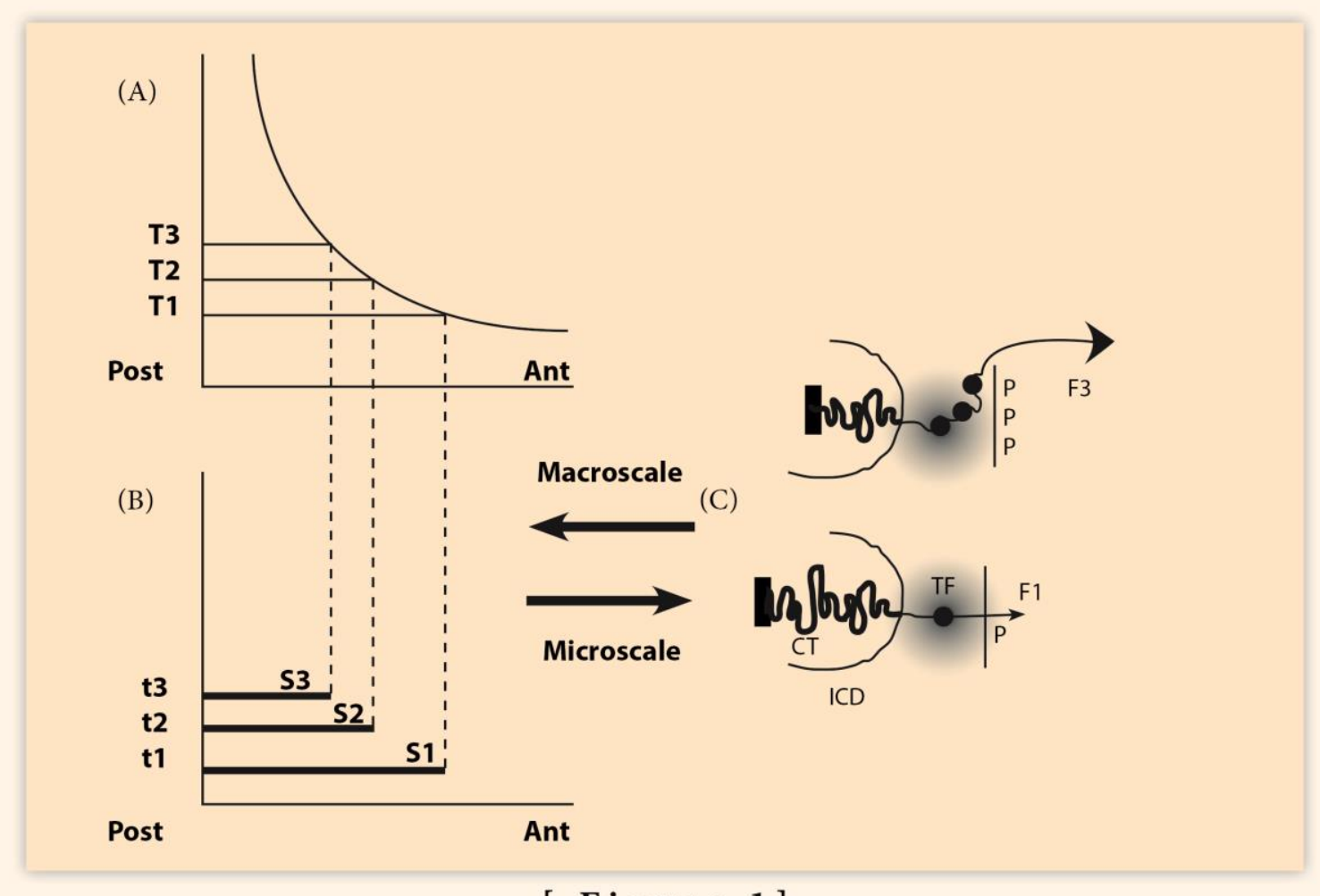

[ Figure 1]

Fig. 1.The macroscale morphogen gradient and the microscale Hox gene clustering in space and time (adapted from Y. Almirantis et al. Current Genomics, 2013, 14 (279-288). (A) Concentrations thresholds (T1, T2, T3) divide the A/P axis in partially overlapping expression domains. (B) The time sequence (t1, t2, t3) combined with the threshold sequence (T1, T2, T3) determine the Hox1, Hox2, Hox3 activation in space and time. S1, S2, S3 are the partially overlapping and nested expression domains of Hox1, Hox2, Hox3. (C) (bottom) In an anterior cell of S1, a small force F1 pulls Hox1 (black spot) out of the chromatin territory (CT) toward the Interchromosome domain (ICD) in the regime of the Transcription Factory (TF) ( grey domain). Allocation of polar molecule $\mathrm{P}$ opposite the telomeric end of the Hox cluster. At a later stage (top), in a more posterior location of S3, a stronger force F3 pulls Hox1, Hox2, Hox3 out of CT in the TF. (Allocation of 3P molecules).

same time technological advances made possible the measurement of geometric modifications of Hox clusters during gene expressions. These modifications include cluster elongations along the 3 ' to 5' direction. Such elongations are naturally attributed to the pulling forces of BM so that the activated Hox clusters behave like irreversibly expanding elastic springs [ 7].

The proper function of an expanding spring depends not only on the pulling forces applied at one of the spring's ends but also on the spring fastening at the other end of the spring. The former action is dynamic while the latter is static. The important role of both actions has been explicitly 
analyzed in [8]. Variations of the pulling forces, for a wide range of forces, are well described by Hooke's empirical law: elongations are proportional to the measure of the pulling forces. As for the role of cluster fastening, any force will slide and expand the spring in accordance with the degree of fastening of HoxA,D [8] (Fig. 2 ). Therefore both dynamic and static physical entities cooperate for the proper function of an elastic spring.

(A)

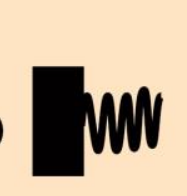

(B)

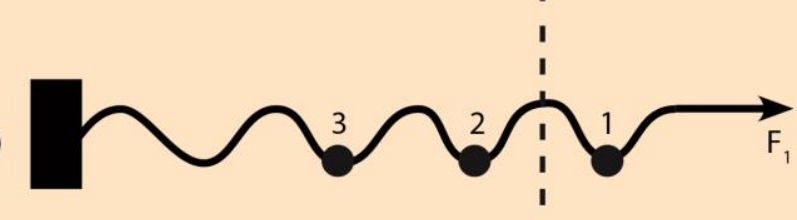

(C)

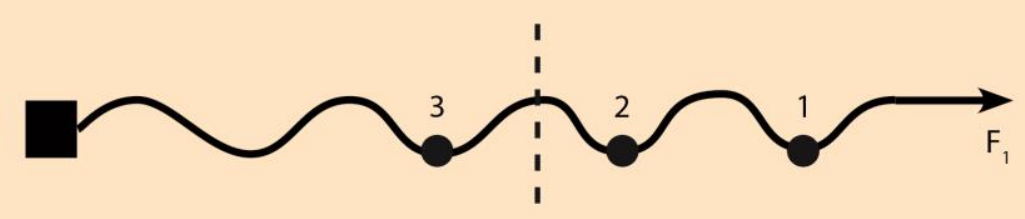

(D)

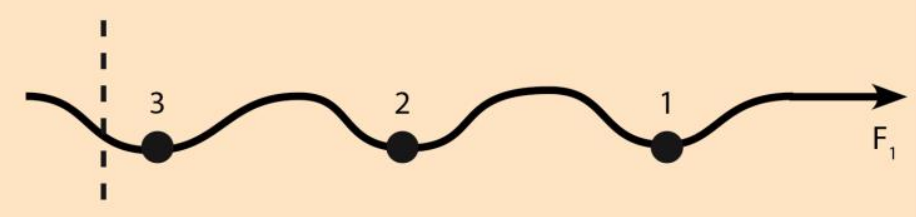

\section{[ Figure 2]}

Fig. 2. Elastic spring expansion under a small pulling force (schematic). From (S. Papageorgiou J. Dev. Biol. 2021, 9(2) 17). (A) In the uncharged spring, no force is applied. The spring is compacted next to the fastening region. (B) A small force F1 is applied to the right end of the spring. The spring fastening is complete (black orthogonal at the left end). The spring expands slightly and a small ball crosses the dashed line to the activation region. (C) The spring fastening is reduced (small black sqare at the left end). Two balls pass to the activation region. (D)The fastening is completely removed and, under the same force F1, all three balls move into the activation region.

\section{Spatial and Temporal Collinearities in the Vertebrates}

\subsection{Paradigm of the HoxA expressions in the chick limb bud.}


At this point it is constructive to examine another paradigm of Hox gene expressions after macroscopic manipulations at the embryonic level as performed in C. Tickle's Laboratory [9] thereafter denoted as (II). In a particular experiment on chick limb buds, this team excised the apical ectodermal ridge (AER) of the bud (II). Then they examined the modified HoxA13 expression in the limb bud. The results are illuminating [9].

1 After the AER excision, HoxA13 is the first gene that rapidly switches off.

2 On a continuous exposure of the limb bud to a FGF soaked bead, HoxA13 is rescued after at least 6 hours.

3 HoxA13 is rescued after 14 hours depending on the dose of FGF soaked bead (higher dose sooner rescue) $[9,10]$.

In the chick limb bud long range action is mainly transmitted by passive diffusion of the morphogen which is produced at the distal end of the limb bud and spreading proximally. At the same time, the morphogen is degradated and finally a steady state morphogen distribution of decreasing exponential form is established with the concentration peak at the distal end [11] (Fig.3). In passive diffusion the velocity of signal propagation is not constant: at the start of diffusion, the spreading velocity is high whereas at later stages it gradually decreases [11]. In Fig.3 a morphogen gradient is depicted where the morphogen source varies. Further analysis is found in (II) .

Tickle and collaborators removed the apical ectodermal ridge (AER) and noticed that after some hours HoxA13 switches off. However, if the FGF soaked beads are persistently inserted distally, the limb bud responds to this insertion and HoxA13 expression is later rescued. However, neither prematurely nor proximally extension of the expression is observed as would be expected according to the morphogen gradient model depicted in Fig.3 [11]. This indicates that the FGF gradient model is necessary but not sufficient for the HoxA expressions in the limb bud (II). Some other complementary mechanisms should be involved for the proper HoxA expressions $[9,10]$. 


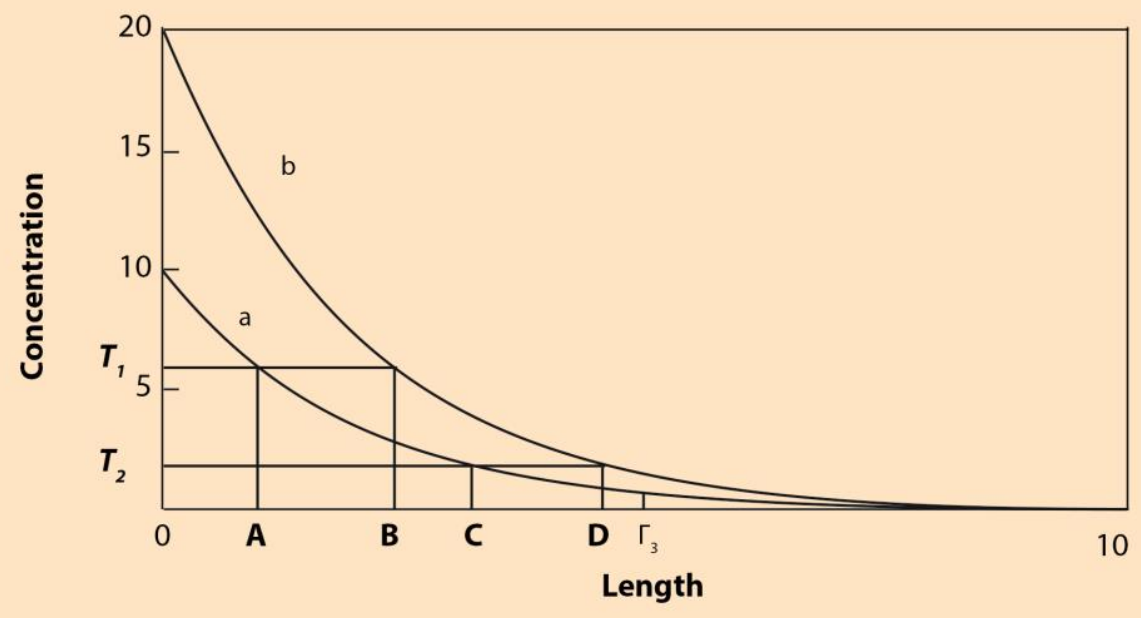

[ Figure 3]

Fig. 3. One-dimensional diffusion gradients in arbitrary units of length and concentration. (From S. Papageorgiou, J Theor Biol , 1998, 192: 43-53). The constant source concentrations at the origin $\mathbf{x}=\mathbf{0}$ are $\mathbf{1 0}$ and $\mathbf{2 0}$ for the curves $\mathbf{a}$ and $\mathbf{b}$ respectively. For every point $\mathbf{x}, \mathbf{b}(\mathbf{x})=\mathbf{2 a}(\mathbf{x})$. This relation is true for any time t $(0 \leq \mathrm{t} \leq \mathrm{t}$ (asymptotic).

The rationale in both paradigms I and II is the same: actions modifying Hox gene expressions are applied in Hox clusters and the resulting consequences are explored. (The common structure and 'identity' of the elastic spring and the Hox cluster is obvious). In Tickle's Lab. the following (Exp. II) was performed in the chick limb.
Exp. II
(a) $\rightarrow($ b)
(b) $\rightarrow$
(c) $\rightarrow$
(d)
(direct step)
(af) $\leftarrow$ (b) $\leftarrow$
(c) $\leftarrow$
(d)
(reverse step) 
According to BM and its elastic spring approximation, state (a) represents the completely fastened spring without any force applied at the right end of the spring (Fig.2A). In (Exp. II) at state (a), the AER is cut-off and substituted by a morphogen FGF bead. At state (b) a force $\mathbf{F}$ applies at the right end of the spring pulling this end of the spring beyond the dashed line (Fig.2B). In state (c) the spring fastening is partly removed so that the spring is shifted further to the right (Fig. 2C). In state (d) the spring fastening is completely removed and the spring is free to slide. Therefore the force $\mathbf{F}$ pulls the whole spring beyond the dashed line (Fig.2D). The final state (d) is a mutant state which is the result of the AER substitution by the FGF bead.

In the reverse step, spring fastening is step by step restored by persistent application of the FGF bead: (d) $\rightarrow$ (af). Note that the final state (af) differs from the starting state (a) since the morphogen gradient model is necessary but not sufficient for the HoxA expressions (see above). This indicates that the substitution of the AER by a morphogen FGF bead is only partly successful since supplementary mechanisms are needed for a complete substitution of the AER [9-11].

\subsection{Paradigm of gene activation in the mouse embryo.}

Next step is to apply the rationale of paradigm II to paradigm I and compare the result. In the direct step of (Exp. I), state (a) of the mouse genome ends up to state (d) where a large posterior upstream DNA domain (including Evx) is cut-off so that, according to (I), TC has disappeared [5].
(Exp. I)
(a) $\rightarrow$ (b) $\rightarrow$
(c) $\rightarrow$
(d)
(direct step)
(a?) $\leftarrow$
(b?) $\leftarrow$
(c?)
(d)
(reverse step)

It will be crucial to test whether the reverse step in (Exp. I) is feasible. This reverse step has not been performed as yet. It is necessary to complete this reverse step in order to properly compare Exp.I and Exp.II.

At this point a divertissement is appropriate. For more than two decades, several groups work intensively in the field of TGF-beta signaling (involving Brachyury expression) in connection with other transcription factors like FGF. In a recent review, it is recognized that Hox gene expressions are interconnected with the Brachyury regulatory gene expression [12]. One pioneering team, including JC Smith from Cambridge, recognized the important role of T-box Brachyury and showed that TGF-beta family signals cause gene spreading by a relay mechanism [13]. Furthermore, this team produced a chromatin imunoprecipitation microarray chip (ChIP- 
chip) which was applied to mouse embryonic stem cells to identify targets of Brachyury [14, 15]. Independently, it was found that more than 500 target genes were identified in zebrafish [12]. These genes respond to signaling like Wnt or FGF [12]. The same signals are involved in both Exp.I and Exp.II. Based on this interconnection, it is a daring hypothesis to assume that the action of the FGF bead in Exp. II and the T-box XBRA action in Exp.I are comparable. To test this hypothesis the reverse step of Exp. I must be completed.

Here it is proposed to apply the above novel microarray chip (ChIP-chip) and search for its effect in the reverse step of (Exp. I). In this reverse step, the mutant state (d) is the starting point where TC disappears according to (I). One cannot foresee what the result will be in the above search (see the following section 4).

\section{Discussion and Conclusions}

A) In the case of paradigm I the chip insertion operates in terra incognita and any prediction should be hazardous. Only Experiment can fix the parameters involved in the data description. Note that an ideal Model is a 'theory' that predicts all experimental results in the realm of its implementation. Usually, a model can predict a limited set of results and all efforts aim at enlarging this domain by experimental trials. In the case of a microarray chip insertion the possible results one can anticipate are the following:

i) no response at all - no difference observed with or without pulling forces.

ii a partial restoration of HoxA13 expression (b?, c?) and

iii) a full rescue of HoxA13 (a?).

Only the experiment will indicate the 'correct' answer.

B) A very important consequence of the above TC disappearance is its connection to TC loss as an evolutionary instrument applied to many animal phyla where TC is absent as, for instance, in Drosophila [8].

C) TC disappearance is a very striking event. A less striking event is TC violation as observed by M. Kondo et al. in Xenopus leavis [16]. These authors analyzed the Allotetraploidization subgenomes during the Whole Genome Duplication and found that some of the two 'homologs' subgenomes (long and short L, S) are not properly aligned in the Hox gene clusters as would be expected according to TC. They concluded therefore that TC may be violated.

D) A general notice could be that usually Experiment precedes Theory and not vice versa: the French Flag was an experimental fact followed by the Positional Information Theory that described it [17]. However in some rare cases this rule is not obeyed, as for example in the genius Turing Theory of Reaction-Diffusion in Morphogenesis. Turing predicted, without any 
preexisting evidence, that specific chemical reactions combined with Diffusion could spontaneously break the symmetric homogeneous state and lead to several inhomogenous patterns as e.g. the pattern formation in embryonic development [17]. This theory of Turing was later experimentally confirmed.

\section{References}

[1] Lewis E.B. A gene complex controlling segmentation in Drosophila. Nature,1978; 276: 565-570.

[2] Krumlauf R. Hox genes, Clusters and Collinearity. Int J Dev Biol, 2018; 62: 659-663.

[3] Lesne A. Systems biology: the multiscale organization of living systems. Medicine Sciences, 2009: 25: 585-587.

[4] Izpisua-Belmonte J.C., Duboule D. Murine genes related to the Drosophila AbdB homeotic genes are sequentially expressed. EMBO J, 1991: 10: 2279-2289.

[5] Kondo T., Duboule D. Breaking Collinearity in the mouse HoxD Complex. Cell, 1999; 97:407-417. Hereafter denoted as [ I]

[6] Papageorgiou S. A physical force may expose Hoxc genes to express in a morphogenetic density gradient. Bull Math Biol, 2001; 63:185-200.

[7] Papageorgiou S. Physical forces may cause the HOXD gene cluster elongation. Biology, 2017, 6(3):32.

[8] Papageorgiou S. Physical Laws shape up HOX gene. J Dev Biol, 2021, 9(2), 17.

[9] Vargesson $\mathrm{N}$ et al. Characterisation of Hoxa gene expression in the chick limb bud in response to FGF. Dev. Dyn. 2001; 220: 87-90. Hereafter denoted as [II].

[10] Towers M. et al. Gradients of signaling in the developing limb. Curr. Opin. Cell Biol. 2012, 24(2):181-187.

[11] Papageorgiou S. Cooperating morphogens control hoxd gene expression in the developing vertebrate limb. J theor Biol, 1998: 192:43-53.

[12] Ye Z, Kimelman D Identification of in vivo Hox13-binding sites reveals an essential locus controlling zebrafish brachyury expression. Development, 2021: 148(11):dev199408. 
[13] Roadway A, et al., Induction of the mesendoderm in the zebrafish germ ring by yolk cellderived TGF-beta family signals and discrimination of mesoderm and endoderm by FGF. Development, 1999, 126(14):3067-78.

[14] Gentsch $\mathrm{G}$ et al. In vivo T-box transcription factor profiling reveals joint regulation of embryonic neuromesodermal bipotency. Cell Reports, 2013: 4(6):1185-96.

[15] Evans A et al. Genomic targets of Brachyury (T) in differentiating mouse embryonic stem cells. PLOS ONE. 2012: 7(3):e33346.

[16] Kondo $\mathrm{M}$ et al. Comprehensive analyses of hox gene expression in Xenopus laevis embryos and adult tissues._Dev Growth Differ. 2017: 59(6):526-539.

[17] Gordon N.K. et al. French flag gradients and Turing reaction-diffusion versus differentiation waves as models of morphogenesis. Biosystems: 2020: 196:104169. 